\title{
Late-week surgery and discharge to specialty care associated with higher costs and longer lengths of stay after elective lumbar laminectomy
}

\author{
Sebastian Salas-Vega, PhD, ${ }^{1}$ Vikram B. Chakravarthy, MD, ${ }^{2}$ Robert D. Winkelman, MD, ${ }^{3}$ \\ Matthew M. Grabowski, MD, ${ }^{2}$ Ghaith Habboub, MD, ${ }^{2}$ Jason W. Savage, MD, ${ }^{2,3}$ \\ Michael P. Steinmetz, MD, ,2,3 and Thomas E. Mroz, MD2,3
}

'London School of Economics and Political Science, London, United Kingdom; 'Department of Neurosurgery, Cleveland Clinic, Cleveland; and ${ }^{3}$ Cleveland Clinic Lerner College of Medicine, Case Western Reserve University, Cleveland, Ohio

\begin{abstract}
OBJECTIVE In a healthcare landscape in which costs increasingly matter, the authors sought to distinguish among the clinical and nonclinical drivers of patient length of stay (LOS) in the hospital following elective lumbar laminectomy-a common spinal surgery that may be reimbursed using bundled payments-and to understand their relationships with patient outcomes and costs.
\end{abstract}

METHODS Patients $\geq 18$ years of age undergoing laminectomy surgery for degenerative lumbar spinal stenosis within the Cleveland Clinic health system between March 1, 2016, and February 1, 2019, were included in this analysis. Generalized linear modeling was used to assess the relationships between the day of surgery, patient discharge disposition, and hospital LOS, while adjusting for underlying patient health risks and other nonclinical factors, including the hospital surgery site and health insurance.

RESULTS A total of 1359 eligible patients were included in the authors' analysis. The mean LOS ranged between 2.01 and 2.47 days for Monday and Friday cases, respectively. The LOS was also notably longer for patients who were ultimately discharged to a skilled nursing facility (SNF) or rehabilitation center. A prolonged LOS occurring later in the week was not associated with greater underlying health risks, yet it nevertheless resulted in greater costs of care: the average total surgical costs for lumbar laminectomy were $20 \%$ greater for Friday cases than for Monday cases, and $24 \%$ greater for late-week cases than for early-week cases ultimately transferred to SNFs or rehabilitation centers. A Poisson generalized linear model fit the data best and showed that the comorbidity burden, surgery at a tertiary care center versus a community hospital, and the incidence of any postoperative complication were associated with significantly longer hospital stays. Discharge to home healthcare, SNFs, or rehabilitation centers, and late-week surgery were significant nonclinical predictors of LOS prolongation, even after adjusting for underlying patient health risks and insurance, with LOSs that were, for instance, 1.55 and 1.61 times longer for patients undergoing their procedure on Thursday and Friday compared to Monday, respectively.

CONCLUSIONS Late-week surgeries are associated with a prolonged LOS, particularly when discharge is to an SNF or rehabilitation center. These findings point to opportunities to lower costs and improve outcomes associated with elective surgical care. Interventions to optimize surgical scheduling and perioperative care coordination could help reduce prolonged LOSs, lower costs, and, ultimately, give service line management personnel greater flexibility over how to use existing resources as they remain ahead of healthcare reforms.

https://thejns.org/doi/abs/10.3171/2020.11.SPINE201403

KEYWORDS laminectomy; neurosurgery; elective surgical procedures; outcome assessment; healthcare costs; length of stay; lumbar

$\mathrm{S}$ URGERIES account for a significant proportion of overall US healthcare expenditures. The total costs per hospitalization have risen with costs per inpatient day, ${ }^{1}$ and surgical admissions now account for $49 \%$ of all inpatient spending. ${ }^{2,3}$ As the healthcare system rapidly evolves in an attempt to manage escalating expenditures and an ever-growing demand, efforts to reduce unnecessarily long hospital lengths of stay (LOSs) may help lower surgical costs and lessen burdens on providers and patients.

Toward this end, recent policy reforms have focused on

ABBREVIATIONS GLM = generalized linear modeling; LOS = length of stay; SNF = skilled nursing facility.

SUBMITTED July 29, 2020. ACCEPTED November 16, 2020.

INCLUDE WHEN CITING Published online April 6, 2021; DOI: 10.3171/2020.11.SPINE201403. 
reducing the LOS with the objective of optimizing resource use. For instance, the 2018 Bundled Payments for Care Improvement (BPCI) Advanced program bundles payments to providers by healthcare episode, providing an incentive to redesign care, reduce the LOS, and control costs. ${ }^{4}$ The program covers 29 inpatient and 3 outpatient clinical episodes, including gastrointestinal, cardiac, orthopedic, and inpatient and outpatient spinal surgical procedures. ${ }^{5}$

To reduce waste rather than merely lower costs, such initiatives must incorporate robust evidence on the factors that influence the duration of hospital stays. For example, advanced age and lower functional class may justify a longer postoperative LOS if that means eventual, safe transfer out of the hospital. ${ }^{6,7}$ On the other hand, the LOS may be equally impacted by factors unrelated to the health needs of patients. In the trauma setting, payer type and discharge destinations independently influence the LOS. ${ }^{8}$ For elective procedures, preoperative factors, including age, sex, and functional status, have been found to affect the LOS in international settings. ${ }^{7}$ Descriptive studies have suggested that the site where surgery is performed and the day of surgery can also prolong the LOS following elective orthopedic procedures. ${ }^{9,10}$ Recent studies have reported similar findings in other surgical specialties, yet they may not adequately adjust for underlying health risks, account for postoperative outcomes and discharge disposition, or evaluate the economic impact from prolonged LOSs. ${ }^{11,12}$ As payment systems change, a failure to reliably distinguish among the clinical and nonclinical predictors of LOS and to understand their relationships with patient outcomes and costs could have a palpable effect on clinical practice and provider reimbursement, and could create adverse incentives for care.

This may be particularly true for elective surgeries. For instance, elective lumbar laminectomy is routinely performed on an inpatient basis to treat symptomatic spinal stenosis. However, the procedure is increasingly performed by outpatient providers - such as ambulatory surgery centers - that may attempt to reduce perioperative surgical costs by limiting patient LOS, ${ }^{13}$ though perhaps at a greater risk of revision surgery and perioperative complications. ${ }^{14}$ In the context of mounting economic pressures to limit the LOS, it is increasingly important to develop an improved understanding of the impact from clinical and nonclinical factors on LOS, patient outcomes, and costs associated with elective surgical procedures.

In the present study, we used regularly collected hospital data to model the impact from clinical and nonclinical factors on patient LOS following elective lumbar laminectomy, a common surgical procedure that can be performed in both inpatient and outpatient settings and can be reimbursed with bundled payments. By offering insights into the factors contributing to prolonged LOSs, as well as their associated health and economic outcomes, this study helps to shed light on opportunities to optimize LOS in elective surgical settings.

\section{Methods}

\section{Patients and Procedure Selection}

A retrospective review was performed for all patients $\geq 18$ years of age who underwent elective lumbar laminectomy for degenerative spinal stenosis between March 1, 2016, and February 1, 2019, in which an open or minimally invasive surgical technique had been applied. Data were obtained from all hospitals in Ohio that are affiliated with the Cleveland Clinic health system.

Degenerative stenosis of the lumbar spine is a common cause of disabling pain in the back and lower extremities that can safely and effectively be treated with surgical decompression via laminectomy. ${ }^{15}$ Lumbar laminectomy is a relatively simple surgical procedure with a largely predictable hospital course. It is also commonly performed: more than 82,550 elective operations were performed in the US in $2012 .{ }^{16}$

\section{Statistical Analysis}

Generalized linear modeling (GLM) was used to examine the relationship between the day of surgery and the patient LOS, our dependent variable. Two candidate models were designed both with and without patient discharge disposition and weekday of surgery; both were adjusted for patient demographics (age, sex, race), weighted Elixhauser comorbidity scores, ${ }^{17}$ incidence of postoperative complications, and other nonclinical factors, including the hospital surgery site and insurance. Discharge planning may be affected by the day of surgery and can impact the patient LOS. One additional model therefore included an interaction term with patient discharge disposition as the mediator variable. Candidate models with the interaction term also considered all seven combinations of demographic variables to adjust for underlying health risks and account for possible overfitting.

Patient discharge disposition included home, home healthcare, and skilled nursing facilities (SNFs) or rehabilitation centers. All insurers covering study-eligible patients were included in our analysis, including private and commercial insurers, Medicare (Advantage and feefor-service), workers' compensation, Medicaid, and others (e.g., self-pay). Our analysis took into account any postoperative complication, including cardiac (acute congestive heart failure, myocardial infarction) and pulmonary (acute respiratory failure, pneumonia) events, as well as altered mental status, durotomy, and infection (Clostridioides difficile, urinary tract infection, or other). Institutions where surgeries were performed were classified as either tertiary care centers or community hospitals, while early- and lateweek surgeries were defined as those occurring on Mondays or Tuesdays and those occurring on Thursdays or Fridays, respectively.

LOS can be represented as a nonzero count variable. GLM models were therefore fitted with Gaussian and Poisson family functions. To account for potential overdispersion of count data, Poisson regression with robust standard errors was used; a third quasi-Poisson family function was also considered. To perform model selection, data were first randomly divided into training $(80 \%)$ and testing (20\%) subsets. A 10-fold cross-validation with a root mean square cost function was used to identify the model that best fit the training data and minimized the cross-validation error term. The best-fitting model was then used to make predictions on test samples and esti- 
mate LOS residuals. A final classifier was then estimated by retraining the best-fitting model on the entire data set.

Total (direct + indirect) healthcare costs associated with each episode of care were obtained from hospital records and adjusted for inflation (2020 US dollars) by using the producer price index for inpatient services from the US Bureau of Labor Statistics. ${ }^{18}$ A chi-square test was used to compare categorical sociodemographic characteristics of individuals undergoing lumbar laminectomy during each day of the week. Patient age, proportion of patients experiencing any postoperative complication, LOS, and adjusted total costs for care were compared across surgical day groups using a one-way ANOVA test. A Wald test was used to assess the significance of model predictor variables. The Nagelkerke pseudo $\mathrm{R}^{2}$ was also used to assess model performance. Statistical significance was defined at the $\mathrm{p} \leq 0.05$ level. All statistical analyses were performed using $\mathrm{R}$ version 3.6.3 (The $\mathrm{R}$ Foundation). This study was reviewed and approved by the institutional review board of the Cleveland Clinic.

\section{Results}

A total of 1359 patients met the eligibility criteria and were included in this study (Table 1). The average age of treated patients varied throughout the workweek and ranged between 64 years (Friday) and 68 years (Tuesday). There was no significant difference among surgical day groups with respect to sex, race, or Elixhauser comorbidity score. ${ }^{17}$

Patients who underwent their procedure on Tuesdays and Wednesdays more frequently received care at a tertiary care center, while those treated on Mondays were more likely to receive care at regional community hospitals. Among all surgical day groups, the greatest proportion of patients were insured by a private or commercial insurance plan, followed by a Medicare fee-for-service plan. There was a statistically significant difference in the proportion of patients covered by each of the insurance plans. Tuesday operations also had the lowest proportion of patients covered by private or commercial insurance plans, while those occurring on Thursdays and Fridays were less likely to be covered by Medicare Advantage and fee-for-service plans.

There was no significant difference in the number of vertebral levels decompressed among the surgical day groups. The incidence of any complication was consistently low, regardless of when the operations took place. There was also no significant difference among surgical day groups in their discharge disposition.

Patients who underwent lumbar laminectomy late in the week had significantly longer hospital LOSs and costs (Table 1). Patients undergoing their procedure between Monday and Friday had mean LOSs of 2.01, 2.04, 2.16, 2.64 , and 2.47 days, respectively. This effect was most notable for patients who were ultimately discharged to SNFs or rehabilitation centers.

Patients who underwent their procedure late in the week were associated with significantly higher total costs for their episodes of care, with average adjusted total costs that were $17.4 \%$ higher for Friday versus Monday cases.
Patients who were discharged to SNFs or rehabilitation centers following treatment late in the week were also associated with an average adjusted total cost that was $21.5 \%$ greater than that for patients who were discharged to SNFs or rehabilitation centers following treatment early in the week. The average surgical costs for late-week surgeries for which patients were discharged to SNFs or rehabilitation centers were also higher among all insurance subgroups.

\section{Regression Analysis}

Of the 27 candidate models that were considered, a Poisson GLM model best fit the data and resulted in the lowest estimate of error. When fitted onto the test sample, the final model had an Akaike information criterion of 855 , a deviance of 134 , and a Nagelkerke pseudo $\mathrm{R}^{2}$ of 0.59 versus the null model. The mean difference between the predicted and observed LOSs also did not significantly differ from zero in any discharge disposition subgroup in which patients underwent surgery early or late in the week.

Predicted LOSs for the test samples were longer for late-week surgery patients who were discharged home or to an SNF or a rehabilitation center, as well as for those who were discharged to home or home healthcare (Fig. 1). However, the difference in the mean predicted LOS among patients undergoing their procedure late versus early in the week was far greater for those discharged to an SNF or a rehabilitation center (1.46 days) than for patients who were discharged to home (0.31 days) or to home healthcare ( 0.46 days).

Retraining the best-fitting Poisson GLM model on the full data set showed that the comorbidity burden, surgery at a tertiary care center versus a community hospital, and incidence of any postoperative complication were independently associated with longer hospital stays (Table 2).

Discharge to home healthcare or to an SNF or a rehabilitation center, in particular, was a significant predictor of a longer LOS, even after adjusting for other covariates, such as underlying health risks and insurance. Patients undergoing their surgical procedure on Thursday or Friday were also found to have a significantly greater likelihood of prolonged postoperative in-hospital stays.

\section{Discussion}

Even as incentives to lower costs and improve patient outcomes evolve, it remains unclear what factors contribute to the hospital LOS following elective surgical procedures. Our study uses regularly collected hospital data to assess the impact from clinical and nonclinical factors, including those related to surgical scheduling, on patient LOS following lumbar laminectomy, a common elective surgical procedure that can be performed in both inpatient and outpatient settings and is reimbursed with bundled payments.

This study finds that the hospital LOS following elective lumbar laminectomy is longer for patients who undergo surgery late in the workweek. Patients treated on Mondays and Tuesdays have an average LOS of 2.01 and 2.04 days, respectively, indicating that their discharge usually occurs by the end of the workweek. In contrast, 
TABLE 1. Patient demographics and sample characteristics

\begin{tabular}{|c|c|c|c|c|c|c|}
\hline & Monday & Tuesday & Wednesday & Thursday & Friday & $\mathrm{p}$ Value \\
\hline No. of patients & 366 & 229 & 279 & 242 & 243 & \\
\hline \multicolumn{7}{|l|}{ Patient characteristics } \\
\hline Age, mean (SD) & $65.40(10.97)$ & $68.04(10.74)$ & $66.13(11.53)$ & $65.22(12.03)$ & $63.70(12.14)$ & 0.001 \\
\hline Male & $243(66.4)$ & $151(65.9)$ & $187(67.0)$ & $149(61.6)$ & $156(64.2)$ & 0.698 \\
\hline Race & & & & & & 0.155 \\
\hline Black & $27(7.4)$ & $10(4.4)$ & $18(6.5)$ & $22(9.1)$ & $9(3.7)$ & \\
\hline Other & $15(4.1)$ & $13(5.7)$ & $14(5.0)$ & $6(2.5)$ & $8(3.3)$ & \\
\hline White & $324(88.5)$ & $206(90.0)$ & $247(88.5)$ & $214(88.4)$ & $226(93.0)$ & \\
\hline Elixhauser score* & & & & & & 0.800 \\
\hline$<0$ & $235(64.2)$ & $139(60.7)$ & $167(59.9)$ & $143(59.1)$ & $141(58.0)$ & \\
\hline 0 & $83(22.7)$ & $54(23.6)$ & $78(28.0)$ & $59(24.4)$ & $62(25.5)$ & \\
\hline $1-4$ & $20(5.5)$ & $13(5.7)$ & $14(5.0)$ & $15(6.2)$ & $12(4.9)$ & \\
\hline$\geq 5$ & $28(7.7)$ & $23(10.0)$ & $20(7.2)$ & $25(10.3)$ & $28(11.5)$ & \\
\hline \multicolumn{7}{|l|}{ Provider \& payer characteristics } \\
\hline Insurance plan & & & & & & 0.041 \\
\hline Private/commercial & $144(39.3)$ & $62(27.1)$ & $101(36.2)$ & $92(38.0)$ & $100(41.2)$ & \\
\hline Medicare Advantage & $75(20.5)$ & $59(25.8)$ & $56(20.1)$ & $44(18.2)$ & $53(21.8)$ & \\
\hline Medicare FFS & $118(32.2)$ & $91(39.7)$ & $104(37.3)$ & $84(34.7)$ & $68(28.0)$ & \\
\hline Workers' comp & $3(0.8)$ & $2(0.9)$ & $5(1.8)$ & $2(0.8)$ & $8(3.3)$ & \\
\hline Medicaid & $22(6.0)$ & $13(5.7)$ & $8(2.9)$ & $14(5.8)$ & $11(4.5)$ & \\
\hline Other & $4(1.1)$ & $2(0.9)$ & $5(1.8)$ & $6(2.5)$ & $3(1.2)$ & \\
\hline \multicolumn{7}{|l|}{ Medical center } \\
\hline Tertiary care & $234(63.9)$ & $192(83.8)$ & $256(91.8)$ & $161(66.5)$ & $188(77.4)$ & $<0.001$ \\
\hline \multicolumn{7}{|l|}{ Procedure \& patient outcomes } \\
\hline Vertebral levels treated, mean (SD) & $1.79(0.96)$ & $1.72(0.93)$ & $1.68(0.98)$ & $1.73(0.90)$ & $1.77(0.96)$ & 0.709 \\
\hline Surgical complication, mean (SD) & $0.13(0.34)$ & $0.13(0.33)$ & $0.13(0.34)$ & $0.16(0.37)$ & $0.14(0.34)$ & 0.832 \\
\hline Discharge disposition & & & & & & 0.696 \\
\hline Home & $274(74.9)$ & $165(72.1)$ & $203(72.8)$ & $172(71.1)$ & $190(78.2)$ & \\
\hline Home healthcare & $61(16.7)$ & $43(18.8)$ & $52(18.6)$ & $42(17.4)$ & $34(14.0)$ & \\
\hline SNF/rehabilitation center & $31(8.5)$ & $21(9.2)$ & $24(8.6)$ & $28(11.6)$ & $19(7.8)$ & \\
\hline LOS, mean (SD) & $2.01(1.83)$ & $2.04(1.89)$ & $2.16(1.89)$ & $2.64(2.76)$ & $2.47(2.43)$ & 0.002 \\
\hline
\end{tabular}

comp = compensation; FFS = fee-for-service.

Values represent the number of patients (\%) unless otherwise indicated.

* Based on Moore et al., 2017.17

the LOS for those treated on Thursdays and Fridays is about 20\%-30\% higher, with average LOSs of 2.64 and 2.47 days, respectively, pushing patient discharge into the weekend. Tuesday operations as well as those occurring on Thursdays and Fridays were less likely to be covered by private or commercial insurance and by Medicare Advantage and fee-for-service plans, respectively. However, this is unlikely to be a direct reflection of insurer preferences. Surgical scheduling often occurs in the clinic after treatment options have been discussed; it involves physicians, patients, and care coordinators, and it is designed to optimize convenience to patients given provider availability. Indeed, late-week surgeries were associated with prolonged LOSs despite there being no significant differences in the number of vertebral levels that were decompressed, the comorbidity burden, or the incidence of postoperative complications among patient groups. These findings also persisted even after adjusting for patient demographics, underlying health risks, hospital surgery site, and insurance.

Discharge disposition also matters. After adjusting for potential confounders, we found that predicted LOSs are longer for patients who are scheduled for late-week surgeries, irrespective of whether they are discharged to home, home healthcare, or SNFs or rehabilitation centers. However, the mean difference in the LOS for earlyversus late-week surgeries is far greater for those being discharged to SNFs or rehabilitation centers (1.46 days) than for those who are discharged to home (0.31 days) or home healthcare (0.46 days). Efforts to reduce unnecessarily long LOSs following elective surgical procedures may therefore have the greatest impact if they target lateweek surgery cases in which the patients are transferred to SNFs or rehabilitation centers. 


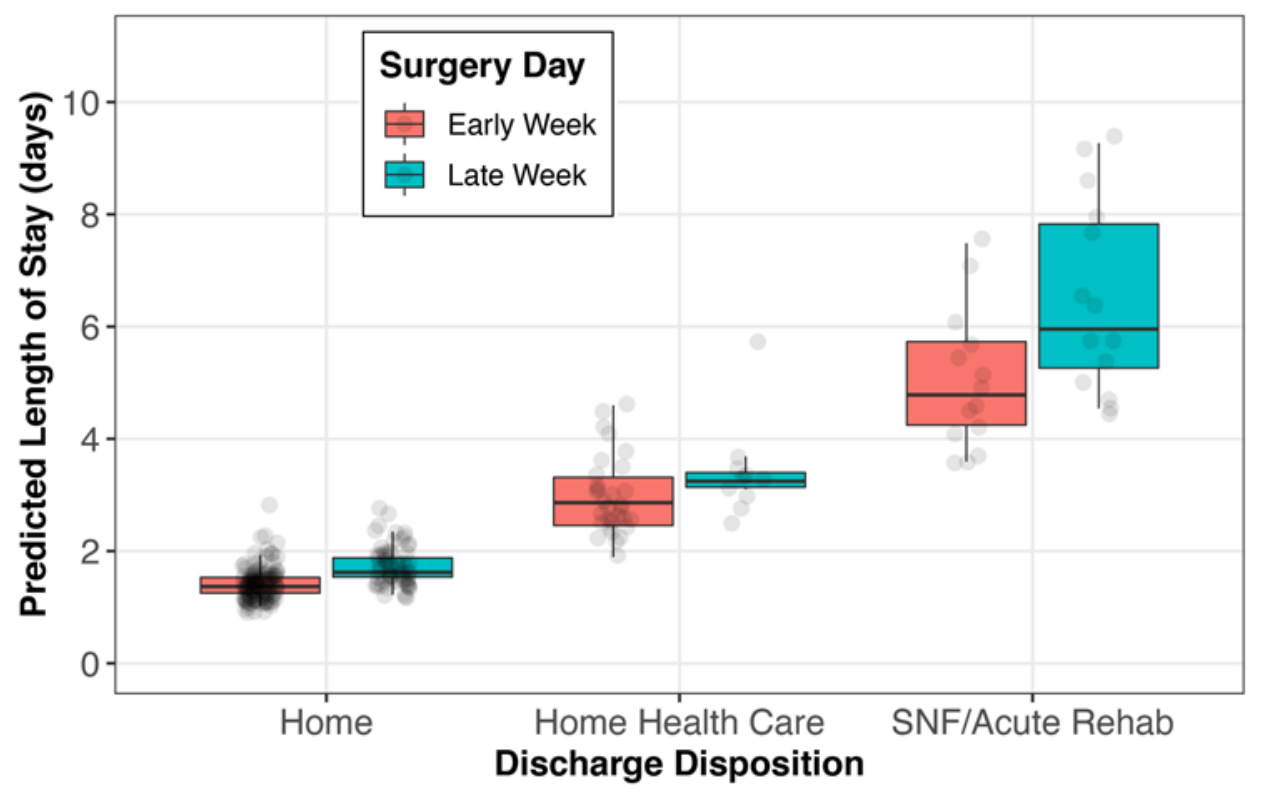

FIG. 1. Box-and-whisker plot depicting predicted lengths of hospital stay with respect to discharge disposition of patients surgically treated early or late in the week. Acute Rehab $=$ acute rehabilitation center; Early Week = Monday + Tuesday + Wednesday; Late Week $=$ Thursday + Friday .

The LOS following elective lumbar laminectomy therefore only partially represents the health needs of patients. Postoperative patient LOSs may instead often be influenced by nonclinical factors, including patient discharge disposition and logistics. Indeed, transfers to SNFs or rehabilitation centers can be delayed by difficulties in coordinating care with insurance companies. ${ }^{19}$ There is also evidence to suggest why such delays in patient discharge may be accentuated over the weekend. For instance, reduced hospital weekend staffing, including fewer occupational and physical therapists and social workers, results in poorer access to ancillary services,$^{20}$ and similar staffing challenges also exist at the subacute skilled nursing and rehabilitation centers that receive patients. ${ }^{21,22}$

Unfortunately, hospital-level data sets, including our own, are not designed to explore the impact from social and logistical factors on postoperative LOSs in great detail. For instance, data sets often lack information on social support, including that from a spouse, as well as information on the auxiliary staff involved in hospital discharge and admission to outside receiving institutions. Future studies should build on this analysis-perhaps by using qualitative or mixed methods - to explore how these factors, including auxiliary staffing at hospitals and specialty care facilities, contribute to LOS among late-week postsurgical patients.

It nevertheless remains the case that late-week surgery for lumbar laminectomy is associated with a longer LOS. This is particularly true for patients who are discharged to skilled nursing or rehabilitation centers. Besides likely worsening patient experience, a prolonged LOS may have adverse financial consequences for payers, providers, and patients. At the extremes, we find that the average total surgical costs for lumbar laminectomy cases performed on Fridays are about $20 \%$ higher than those for cases that take place on Mondays. The average total surgical cost associated with patients discharged to an SNF or rehabilitation center late in the week was also $24 \%$ greater than those associated with patients treated early in the week. Average surgical costs were also higher for all insurance types, pointing to potential cost savings to providers irrespective of payer. Without any clear evidence of differences in pre- or postoperative health needs, these findings suggest that prolonged LOSs among late-week surgery cases, particularly those transferring to SNFs or rehabilitation centers, contribute to unnecessary and potentially avoidable healthcare costs.

At the same time, we find little evidence to suggest that prolonged postoperative LOSs improve health outcomes. Our study finds that the incidence of complications following lumbar laminectomy is low and unrelated to the weekday on which the procedure takes place. The likelihood of patient discharge to SNFs or rehabilitation centers also did not differ across surgical day groups.

That said, another limitation of our study is that we cannot assess the impact from prolonged LOS and the potentially greater intensity of in-hospital care on health outcomes occurring after discharge. In the cardiac literature, admission to a hospital for myocardial infarction over the weekend and during evenings is associated with significantly greater odds of 30-day mortality. ${ }^{23}$ In our situation, while one could reasonably hypothesize that extended stays in the hospital following elective lumbar laminectomy would reduce the risk of some complications occurring in the postdischarge setting, it could also increase the risk of other complications, such as nosocomial infection. While further research is needed to evaluate the long-term consequences of prolonged LOS, our study finds no evidence to support the notion that patients fare better from a prolonged LOS while they remain in the hospital. 
TABLE 2. Poisson regression-optimized model

\begin{tabular}{|c|c|}
\hline & Poisson Regression-LOS \\
\hline Intercept & $3.00^{*}(1.91,4.70)$ \\
\hline Age & $0.96(0.85,1.09)$ \\
\hline \multicolumn{2}{|l|}{ Sex } \\
\hline Male & $0.86(0.71,1.04)$ \\
\hline \multicolumn{2}{|l|}{ Race } \\
\hline Other & $0.73(0.44,1.20)$ \\
\hline White & $0.78(0.53,1.15)$ \\
\hline \multicolumn{2}{|c|}{ Center where surgery was performed } \\
\hline Tertiary care & $1.33 \dagger(1.05,1.68)$ \\
\hline \multicolumn{2}{|l|}{ Insurance } \\
\hline Medicare HMO & $1.21(0.88,1.68)$ \\
\hline Medicare FFS & $0.81(0.64,1.02)$ \\
\hline Workers' comp & $0.81(0.52,1.28)$ \\
\hline Medicaid & $1.30(0.89,1.90)$ \\
\hline Other & $1.41(0.63,3.18)$ \\
\hline \multicolumn{2}{|l|}{ Elixhauser scoref } \\
\hline$<0$ & $1.42^{*}(1.22,1.66)$ \\
\hline $1-4$ & $1.50(0.96,2.33)$ \\
\hline$\geq 5$ & $2.36^{*}(1.53,3.63)$ \\
\hline \multicolumn{2}{|l|}{ Surgical complication } \\
\hline Any & $3.31^{*}(2.30,4.75)$ \\
\hline \multicolumn{2}{|l|}{ Discharge disposition } \\
\hline Home healthcare & $4.83^{*}(3.69,6.31)$ \\
\hline SNF/rehabilitation center & $49.58^{*}(26.86,91.52)$ \\
\hline \multicolumn{2}{|c|}{ Day when surgery was performed } \\
\hline Tuesday & $0.94(0.73,1.21)$ \\
\hline Wednesday & $1.09(0.86,1.37)$ \\
\hline Thursday & $1.55 \S(1.14,2.12)$ \\
\hline Friday & $1.61^{*}(1.23,2.11)$ \\
\hline No. of patients & 1359 \\
\hline $\mathrm{AIC}$ & 5202.21 \\
\hline $\mathrm{BIC}$ & 5316.93 \\
\hline Pseudo R² & 0.45 \\
\hline \multicolumn{2}{|l|}{$\begin{array}{l}\text { AIC = Akaike information criterion } \\
=\text { health maintenance organizatio } \\
\text { Values are expressed as exponen } \\
\text { limits). All continuous predictors a } \\
\text { deviation. Standard errors are het } \\
{ }^{*} p<0.001 . \\
\dagger p<0.05 . \\
\ddagger \text { Based on Moore et al., } 2017.17 \\
\S p<0.01 .\end{array}$} \\
\hline
\end{tabular}

Moreover, our study is based on data from a single healthcare system. While the Cleveland Clinic is one of the largest academic hospital systems in the United Stateswith 18 hospitals in northeast Ohio alone-idiosyncrasies in the patient case mix or healthcare delivery may limit this study's generalizability to other hospital systems. Additional studies should attempt to replicate this analysis in other settings to further explore this issue.

In summary, findings from our study suggest that hos- pital LOSs following elective spine procedures are prolonged by clinical and nonclinical factors, including the day of surgery and the discharge disposition. These findings point to opportunities to optimize the costs and outcomes of elective surgical care. For instance, our research group is studying the use of various technologies, including machine learning, to automate surgical scheduling and other clinical processes based in part on expectations of postoperative health needs. Such an approach may help identify surgical patients who are at greatest risk of requiring transfer to an SNF or rehabilitation center, allowing providers to front-load these cases earlier in the week. Doing so may initially be disruptive to clinical operations, particularly considering surgical block time allocations. However, it could also help reduce prolonged LOSs, lower costs for patients and payers, allow providers to make more efficient use of surgical resources, and, ultimately, give service line management personnel greater flexibility over how to use existing resources as they remain ahead of healthcare reforms. In a healthcare landscape in which costs increasingly matter, such initiatives in surgical care are a worthwhile endeavor.

\section{Conclusions}

Hospital LOS following elective lumbar laminectomy is longer for patients who undergo surgery late in the workweek, despite there being little evidence to indicate greater health needs among these patients. This effect appears to be particularly true for patients who are ultimately discharged to an SNF or rehabilitation center. Indeed, after adjusting for potential confounders, predicted LOSs are greater for patients who are scheduled for late-week surgeries irrespective of discharge disposition. However, the mean difference in LOS for early- versus late-week surgeries is far greater for those discharged to SNFs or rehabilitation centers (1.46 days) than for those who are discharged to home ( 0.31 days) or home healthcare $(0.46$ days). Prolonged LOS does not appear to result in improved in-hospital patient outcomes. It nevertheless results in greater costs of care, with average total surgical costs for lumbar laminectomy that are $20 \%$ greater for Friday cases than for Monday cases and 24\% greater for late- versus early-week cases that ultimately transfer to SNFs or rehabilitation centers.

\section{Future Directions}

Our study points to opportunities to lower costs and improve outcomes associated with elective surgical care, notably by optimizing surgical scheduling and perioperative care coordination. The use of predictive analytics during the surgical scheduling process could, for instance, help identify spine surgery patients at greatest risk of requiring transfer to an SNF or rehabilitation center, allowing providers to schedule these cases earlier in the week. Such an approach may reduce prolonged hospital LOS, lower costs for patients and payers, allow providers to make more efficient use of surgical resources, and, ultimately, give service line management personnel greater flexibility over how to use existing resources as they remain ahead of healthcare reforms. 


\section{Acknowledgments}

We thank Nicolas Thompson for his feedback. Publication of this work was graciously supported by grants to Dr. Salas-Vega from the Sanford J. Larson Honor Your Mentor Fund and the Academy of Medicine Education Foundation.

\section{References}

1. Healthcare Cost and Utilization Project (HCUP). HCUP Fast Stats-Trends in inpatient stays. Agency for Healthcare Research and Quality. Accessed December 29, 2020. http:// www.hcup-us.ahrq.gov/faststats/national/inpatienttrends.jsp

2. 2018 Health Care Cost and Utilization Report. Health Care Cost Institute; 2020. Accessed December 29, 2020. https:// healthcostinstitute.org/images/pdfs/HCCI_2018_Health_ Care_Cost_and_Utilization_Report.pdf

3. Muñoz E, Muñoz W III, Wise L. National and surgical health care expenditures, 2005-2025. Ann Surg. 2010;251(2):195-200.

4. Joynt Maddox KE, Orav EJ, Zheng J, Epstein AM. Post-acute care after joint replacement in Medicare's bundled Payments for Care Improvement initiative. J Am Geriatr Soc. 2019; 67(5):1027-1035.

5. BPCI Advanced: General frequently asked questions (FAQ). Centers for Medicare \& Medicaid Services (CMS). Accessed December 29, 2020. https://innovation.cms.gov/files/x/bpciadvanced-faqs.pdf

6. Collins TC, Daley J, Henderson WH, Khuri SF. Risk factors for prolonged length of stay after major elective surgery. Ann Surg. 1999;230(2):251-259.

7. Lee SY, Lee SH, Tan JHH, et al. Factors associated with prolonged length of stay for elective hepatobiliary and neurosurgery patients: a retrospective medical record review. $B M C$ Health Serv Res. 2018;18(1):5.

8. Brasel KJ, Lim HJ, Nirula R, Weigelt JA. Length of stay: an appropriate quality measure? Arch Surg. 2007;142(5):461-466.

9. Keswani A, Beck C, Meier KM, et al. Day of surgery and surgical start time affect hospital length of stay after total hip arthroplasty. J Arthroplasty. 2016;31(11):2426-2431.

10. Martino J, Peterson B, Thompson S, et al. Day of week and surgery location effects on stay length and cost for total joint arthroplasty: academic versus orthopaedic-specific hospital. J Knee Surg. 2018;31(9):815-821.

11. Hijji FY, Narain AS, Haws BE, et al. Does day of surgery affect hospital length of stay and charges following minimally invasive transforaminal lumbar interbody fusion? Clin Spine Surg. 2018;31(5):E291-E295.

12. Sivaganesan A, Devin CJ, Khan I, et al. Is length of stay influenced by the weekday on which lumbar surgery is performed? Neurosurgery. 2019;85(4):494-499.

13. Makanji HS, Bilolikar VK, Goyal DKC, Kurd MF. Ambulatory surgery center payment models: current trends and future directions. J Spine Surg. 2019;5(suppl 2):S191-S194.

14. Arshi A, Wang C, Park HY, et al. Ambulatory anterior cervical discectomy and fusion is associated with a higher risk of revision surgery and perioperative complications: an analysis of a large nationwide database. Spine J. 2018;18(7): $1180-1187$.

15. Hilibrand AS, Rand N. Degenerative lumbar stenosis: diagnosis and management. J Am Acad Orthop Surg. 1999;7(4): $239-249$
16. Bernstein DN, Brodell D, Li Y, et al. Impact of the economic downturn on elective lumbar spine surgery in the United States: a national trend analysis, 2003 to 2013. Global Spine J. 2017;7(3):213-219.

17. Moore BJ, White S, Washington R, et al. Identifying increased risk of readmission and in-hospital mortality using hospital administrative data: the AHRQ Elixhauser Comorbidity Index. Med Care. 2017;55(7):698-705.

18. Dunn A, Grosse SD, Zuvekas SH. Adjusting health expenditures for inflation: a review of measures for health services research in the United States. Health Serv Res. 2018;53(1): 175-196.

19. Sorensen M, Sercy E, Salottolo K, et al. The effect of discharge destination and primary insurance provider on hospital discharge delays among patients with traumatic brain injury: a multicenter study of 1,543 patients. Patient Saf Surg. 2020;14(1):2.

20. Hendy P, Patel JH, Kordbacheh T, et al. In-depth analysis of delays to patient discharge: a metropolitan teaching hospital experience. Clin Med (Lond). 2012;12(4):320-323.

21. Weinberg AD, Lesesne AJ, Richards CL, Pals JK. Quality care indicators and staffing levels in a nursing facility subacute unit. J Am Med Dir Assoc. 2002;3(1):1-4.

22. Geng F, Stevenson DG, Grabowski DC. Daily nursing home staffing levels highly variable, often below CMS expectations. Health Aff (Millwood). 2019;38(7):1095-1100.

23. Jayawardana S, Salas-Vega S, Cornehl F, et al. The relationship between off-hours admissions for primary percutaneous coronary intervention, door-to-balloon time and mortality for patients with ST-elevation myocardial infarction in England: a registry-based prospective national cohort study. BMJ Qual Saf. 2020;29(7):541-549.

\section{Disclosures}

Dr. Savage reports a consultant relationship with Stryker. Dr. Steinmetz reports a consultant relationship with Globus; receipt of speaker's fees from Zimmer Biomet, Globus, and Elsevier; and receipt of honoraria from Globus and Stryker. Dr. Mroz reports receipt of royalties from Stryker.

\section{Author Contributions}

Conception and design: Salas-Vega, Winkelman, Grabowski. Acquisition of data: Salas-Vega, Winkelman, Habboub. Analysis and interpretation of data: Salas-Vega, Winkelman, Habboub. Drafting the article: Salas-Vega, Chakravarthy, Winkelman. Critically revising the article: Salas-Vega, Chakravarthy, Savage, Steinmetz, Mroz. Reviewed submitted version of manuscript: all authors. Approved the final version of the manuscript on behalf of all authors: Salas-Vega. Statistical analysis: Salas-Vega, Winkelman. Administrative/technical/material support: SalasVega, Habboub, Savage, Steinmetz, Mroz. Study supervision: Salas-Vega, Grabowski, Habboub, Savage, Steinmetz, Mroz.

\section{Correspondence}

Sebastian Salas-Vega: London School of Economics and Political Science, London, UK. s.salas-vega@lse.ac.uk. 